\title{
Serum Pharmacochemistry Analysis Combined with Network Pharmacology Approach to Investigate the Antiosteoporosis Effect of Xianlinggubao Capsule in vivo
}

\author{
Yun-Hui Xu ${ }^{1 \#}$ Yi-Chun Sun ${ }^{2,3 \#}$ \\ Tong $\mathrm{Wu}^{1 *}$ Xiao $\mathrm{Hu}^{1,2 *}$
}

Address for correspondence Xiao Hu, Shanghai Institute of Pharmaceutical Industry, 285 Gebaini Road, Shanghai 201203, People's Republic of China (e-mail: xjtuyxhx@126.com).

Tong Wu, Shanghai Institute of Pharmaceutical Industry, 285 Gebaini Road, Shanghai 201203, People's Republic of China (e-mail: tongwu88@163.com).

Pharmaceut Fronts 2020;2:e168-e178.

\begin{abstract}
Xianlinggubao capsule (XLGB) is a traditional Chinese medicine multi-component herbal prescription and has been widely used in osteoporosis (OP) treatment. However, the underlying anti-OP mechanisms of XLGB have not been fully studied. In this study, an ovariectomized rat model of OP was established. The OP rats were orally administrated with XLGB, and then the main absorbed components in serum sample were assessed based on liquid chromatography-tandem mass spectrometry

Keywords

- xianlinggubao capsule

- osteoporosis

- mechanism

- serum pharmacochemistry analysis

- network pharmacology

- molecular docking (LC-MS/MS). Subsequently, the potential anti-OP markers in XLGB were screened based on a network pharmacology strategy. Molecular docking analysis was used for confirmation. LC-MS showed 22 absorbed components in the serum sample of OP rat with XLGB treatment. Network pharmacology and pathway analysis suggested 19 potential anti-OP markers in XLGB. According to molecular docking process, most of the potential markers displayed strong interactions with the 22 absorbed components mentioned above. Besides, an absorbed component-potential marker-pathway network was further established. In conclusion, our data suggested the possible mechanisms for XLGB in OP treatment, in which the "multicomponents, multitargets, and multipathways" participated. Our article provided possible direction for drug discovery in OP and could help for exploring novel application of XLGB in clinical setting.
\end{abstract}

\section{Introduction}

Osteoporosis (OP) is a complex bone disorder with a genetic background, and is characterized by progressive deterioration in bone tissue, which results in a decrease in bone mass, quality, and strength, and an increase in bone fracture

\# These authors contributed equally to this work.

received

December 28, 2020

accepted

February 10, 2021

published online

April 8, 2021 risk. $^{1,2}$ It has been estimated that the number of annual OPrelated fractures in the United States will increase from 2 to 3 million in the period from 2005 to 2025, and the associated acute and long-term medical care cost will increase from $\$ 17$ billion to $\$ 25$ billion. ${ }^{3}$ The prevalence and high medical costs of OP also remain true in developing countries including China. According to epidemiological investigation, China's OP incidence rate in 2008 was 14.94\%; however, during 2012 to

\section{(c) 2021. The Author(s).}

This is an open access article published by Thieme under the terms of the Creative Commons Attribution License, permitting unrestricted use, distribution, and reproduction so long as the original work is properly cited. (https://creativecommons.org/licenses/by/4.0/)

Georg Thieme Verlag KG, Rüdigerstraße 14, 70469 Stuttgart, Germany
DOI https://doi.org/ $10.1055 / \mathrm{s}-0041-1726301$. ISSN 2628-5088. 
2015 , it increased up to $27.96 \%,{ }^{4}$ with, the direct medical costs of hip fracture in 2006 reaching approximately US $\$ 1.5$ billion. ${ }^{5}$ It is obvious that with the rapid increase in aging population, OP incidence in developing countries will increase more and more significantly in the future. Evidence suggests that by 2050, the number of hip fractured in Asia will be increase by $50 \%{ }^{6,7}$ Given above, OP is a growing global public health problem with substantial medical, social, and economic burden.

Chinese herbal medicines play an important role in the preclinical and clinical studies of $\mathrm{OP}^{8}$ Xianlinggubao formula was designed based on modification of the empirical "Miao minority" medicine, which was commonly used to tone the "kidney system" and nourish bones. Xianlinggubao capsule (XLGB) was officially approved and sold as the over-the-counter (OTC) drug in China (CFDA, Z20025337). It is the only traditional Chinese medicine (TCM) prescription that was used for the prevention and treatment of OP on the National Health Insurance List in China. Its safety and efficacy have been proven by the principle of evidence-based medicine. ${ }^{9}$ Preclinical studies showed that XLGB could improve bone mineral density and mechanical strength in ovariectomized (OVX)-induced OP in old rats. ${ }^{10}$ However, the active compounds and the underlying mechanism of XLGB have not been fully understood.

As a TCM Fufang (multi-component) prescription, XLGB consists of six commonly used Chinese herbs with their percentages in weight as follows: Herba Epimedii (Epimedium brevicornu Maxim, Yinyanghuo) (70\%), Radix Dipsaci (root of Dipsacus asper Wall ex Henry, Xuduan) (10\%), Rhizoma Anemarrhenae (rhizome of Anemarrhena asphodeloides Bunge, Zhimu) (5\%), Radix et Rhizoma Salviae (root and rhizome of Salvia miltiorrhiza Bunge, Danshen) (5\%), Fructus Psoraleae (fruit of Psoralea corylifolia L., Buguzhi) (5\%), and Radix Rehmanniae (root of Rehmannia glutinosa Libosch, Dihuang) (5\%). ${ }^{11}$ Chemical analysis showed that there are a large number of compounds with different structures in this formula, ${ }^{12}$ which makes the study of the anti-OP mechanism of XLGB a great challenge. In 2020, Bao et al used the network pharmacology, a popular method for investigating the underlying molecular mechanisms for TCM in recent years, to systematically study the possible therapeutic mechanisms of XLGB in OP. ${ }^{13,14}$ It is well known that XLGB, an oral medicine, plays roles only if it is absorbed into the blood, thus, investigating the anti-OP mechanisms of XLGB at the molecular and system levels in vivo remains urgent.

In this study, we identified absorbed compounds of XLGB in the serum sample of OVX rats through a pharmacochemistry test. Potential markers were screened by network pharmacology analysis based on protein-protein interaction (PPI) data and KEGG (Kyoto Encyclopedia of Genes and Genomes) enrichment. Molecular docking was employed to investigate the strength of interaction between absorbed components and potential markers. Furthermore, an absorbed componentpotential marker-pathway network was established. The detailed flowchart of this work is depicted in - Fig. 1. Our data first and systematically demonstrated the therapeutic mechanisms XLGB in an in vivo study.

\section{Materials and Methods}

\section{Serum Pharmacochemistry Analysis of XLGB}

Establishment and Treatment of OVX-Induced OP in Rats Female Sprague-Dawley rats (200-220 g) were anesthetized with pentobarbital sodium $(40 \mathrm{mg} / \mathrm{kg}$, intraperitoneal), and their ovaries were removed bilaterally. Thirty days later, the indexes of bone density were measured by X-ray absorptiometry. Serum concentrations of osteocalcin, N-terminal propeptide of type I procollagen (PINP), alkaline phosphatase (ALP), and tartrate resistant acid phosphatase (TRAP) were estimated using an enzyme-linked immunosorbent assay kit following the reported study to assess the successful establishment of OVX-induced OP in rat models. ${ }^{15-17}$

OVX rats were divided into two groups: XLGB and control groups. Rats in the XLGB group $(n=5)$ were intragastrically treated with XLGB $(300 \mathrm{mg} / \mathrm{kg}$ daily, Lot No. 1611016 , GuizhouTongji Tang Pharmaceutical Co., Ltd.) for 50 consecutive days. Rats in the control group $(n=5)$ were treated with physiological saline in the same way as that in the XLGB group. All experiments were approved by the Laboratory Animal

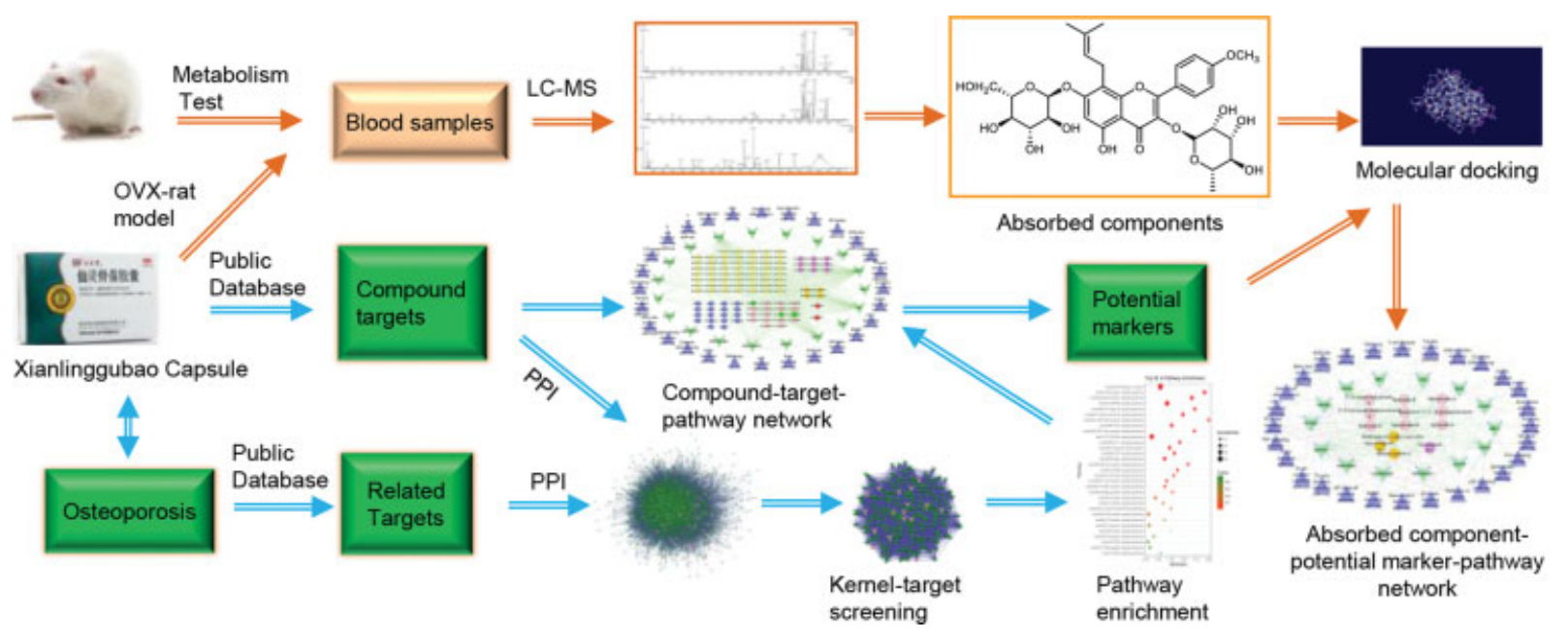

Fig. 1 The flowchart of network pharmacology approach. 
Ethics Committee of Shanghai Institute of Pharmaceutical Industry.

\section{Identification of the Absorbed Components}

On the 50th day, all rats were anesthetized by intraperitoneal injection of $10 \%$ aqueous chloral hydrate. After 2 hours, the blood samples from the hepatic portal vein were collected, centrifuged at $14,000 \mathrm{rpm}$ for 15 minutes at $4^{\circ} \mathrm{C}$, and then mixed to produce the pooled plasma.

Serum pharmacochemistry analysis was performed on a Waters UPLC (ultra-performance liquid chromatography) system using an ACQUITY UPLCHSS T3 column $(2.1 \times 100 \mathrm{~mm}$, $1.7 \mu \mathrm{m}$ ) with a security guard column of the same material at a flow rate of $0.4 \mathrm{~mL} /$ minute. The mobile phases $A$ and $B$ were $0.1 \%$ formic acid in water and acetonitrile, respectively. The gradient program was as follows: 0-0.15 minutes, $98 \% \mathrm{~A}$; 0.15-17.34 minutes, $98-69 \% \quad A ; \quad 17.34-20.78$ minutes, 69-52\% A; 20.78-24.22 minutes, 52-20\% A; 24.2226.00 minutes, 20-0\% A, 26.00-29.00 minutes, 0\% A; 29.0029.10 minutes, $0-2 \%$ A; 32.5 minutes, $2 \%$ A. UV spectra from 190 to $400 \mathrm{~nm}$ were recorded online.

Mass spectra were acquired in both positive and negative ion modes by using a Waters definition accurate mass quadrupole time-of-flight (Q-TOF) Xevo G2-XS mass spectrometer (Waters MS Technologies, United Kingdom) equipped with an electrospray ionization source. The optimized operating parameters were set as follows: mass range, $m / z$ : $50-1,500$; the flow rate of drying gas $\left(\mathrm{N}_{2}\right): 800 \mathrm{~L} / \mathrm{h}$; drying gas temperature: $400^{\circ} \mathrm{C}$; cone gas flow: $100 \mathrm{~L} / \mathrm{h}$; source temperature: $120^{\circ} \mathrm{C}$; capillary voltage: $2.5 \mathrm{kV}$; cone voltage: $40 \mathrm{~V}$; in MSE mode, the energies for collision induced dissociation were $6 \mathrm{~V}$ for the precursor ion at low energy mode and 30 to $60 \mathrm{~V}$ for fragmentation information at high energy mode. An external reference (Lock-Spray) consisting of a $0.2 \mathrm{ng} / \mathrm{mL}$ solution of leucine enkephalin was used in both positive $\left(m / z: 556.2771[\mathrm{M}+\mathrm{H}]^{+}\right)$and negative modes $\left(\mathrm{m} / \mathrm{z}: 554.2615[\mathrm{M}-\mathrm{H}]^{-}\right)$, infused at a flow of $5 \mu \mathrm{L} /$ minute. All the data were acquired using MassLynx 4.1 software (Waters, Milford, Massachusetts, United States).

\section{Potential Marker Screening}

Composite Compounds and ADME (Absorption,

Distribution, Metabolism, Excretion) Screening of XLGB

The chemical composition of all the six herbs of XLGB was obtained from the Traditional Chinese Medicine System Pharmacology Database (TCMSP, http://lsp.nwu.edu.cn/ tcmsp.php) and TCM database@Taiwan (http://tcm.cmu. edu.tw/index.php). Pharmacokinetic properties of each compound, including the prediction of oral bioavailability (OB), intestinal epithelial permeability (Caco-2 cells), drug likeness (DL), blood-brain barrier (BBB), drug half-life, and Lipinski's rule (LR) of five ${ }^{18}$ were assessed for screening and evaluation of the target compounds. The selected candidate molecules should satisfy the criteria: $\mathrm{OB} \geq 30 \%, \mathrm{DL} \geq 0.18$, Caco- $2>-0.40$, suggested by the TCMSP database ${ }^{19}$ and LR of five. Besides, to discover the active ingredients as much as possible, we searched a large number of texts and selected some main ingredients of these herbs or compounds with pharmacological activity in our prescreening process to supplement the compound library.

\section{Compound Targets for XLGB}

The potential targets of the predicted active compounds of XLGB were explored from TCMSP. Besides, SWISS database (http://www.swisstargetprediction.ch), STITCH database (http://stitch.embl.de), and target prediction system (https://prediction.charite.de/subpages/target_prediction. php) were further used for prediction for those that failed to be collected in TCMSP. UniProt (http://www.uniprot.org/) serves as a central hub for the collection of functional information on proteins, and has the advantages of accurate, consistent, and rich annotation. In this study, UniProt was applied to resolve nonstandard naming.

\section{Candidate Targets for OP}

OP-related targets were collected using "Osteoporosis" as the keyword from four existing resources, namely, Online Mendelian Inheritance in Man database (OMIM; http://www. omim.org/), Genetic Association Database (GAD; http:// geneticassociationdb.nih.gov/), Therapeutic Target Database (TTD; http://db.idrblab.net/ttd/), and pharm-GKB (https:// www.pharmgkb.org/). The official gene symbols were acquired through UniProt translation.

\section{Kernel Targets for XLGB against OP}

System biology studies show that genes and proteins are interconnected and the PPI networks are usually used to understand the role of various proteins in complex diseases. PPI data can be obtained using the Cytoscape plugin Bisogenet. In this study, the intersection of compound target PPI data and OP target PPI data was established to study the antiOP effect of compounds, and the kernel targets were screened with node degree and Cytoscape plugin CytoNCA.

\section{Enrichment Analysis and Potential Target Screening}

The Database for Annotation, Visualization and Integrated Discovery (DAVID; https://david-d.ncifcrf.gov, ver. 6.8) was applied. $^{20}$ Pathway enrichment was performed through inputting the kernel genes into DAVID.

\section{Molecular Docking}

The structure of the absorbed compounds was downloaded from PubChem (https://pubchem.ncbi.nlm.nih.gov/). In this study, three-dimensional structures of the 19 target peptides were derived from the RCSB Protein Data Bank (http://www.rcsb.org/pdb/home/home.do). The docking calculation was performed using the software DOCK (6.7) on Yinfo Cloud Computing Platform, a 6 server for biomedicinal, material, and statistical researches (http://cloud. yinfotek.com). ${ }^{21}$ The best ranked docking pose of the peptide in the active site of each compound was determined according to the grid scores.

\section{Network Construction}

All the networks can be created via utilizing the network visualization software Cytoscape (http://cytoscape.org/, ver. 
3.4.0). ${ }^{22}$ This is a software that applies to visualizing biological pathways, intermolecular interaction networks, etc. Meanwhile, it also supplies a basic set of features for data integration, analysis, and visualization for complicated network analysis.

\section{Results}

\section{Pharmacochemistry Analysis of XLGB in Serum Sample of OP Rats}

Successful Establishment of OVX Model of OP in Rats In this article, the OVX-induced OP model in rat was established. - Fig. 2 shows that bone density and osteocalcin in model rats decreased significantly ( $p<0.05$ and $p<0.01$ vs. normal, respectively), while serum levels of PINP, ALP, and TRAP increased significantly ( $p<0.01$ vs. normal), demonstrating the successful establishment of the OVX model of OP in rats.

\section{Identification of the Absorbed Components}

According to the UPLC-QTOF MS analysis, 22 prototype components were identified from OVX rat plasma after oral administration of XLGB (-Fig. 3 and -Table $\mathbf{1}$ ).

\section{Potential Target Screening}

\section{Candidate Compound Screening for XLGB}

A total of 520 ingredients were obtained from TCMSP, and 83 candidate compounds were retained after LR and ADME screening; 28 compounds of Fructus Psoraleae were gained from TCM database@Taiwan. In addition, 15 compounds, either with extensive pharmacological activities or were the typical components of six herbs but failed to meet LR or the ADME criteria, have been supplemented. In total, 126 candidate compounds were determined and are listed in -Supplementary Table S1 (online only).

Identification of Common Targets for XLGB against OP A total of 328 putative targets for 124 candidate compounds were collected from the aforementioned database, while 285 OP-related targets were collected, of which 94, 215, 10, and 24 from OMIM, GAD, pharm-GKB, and TTD, respectively.

\section{Pathway Enrichment for Kernel Targets and Potential Marker Screening}

First, an intersected network was constructed between a compound putative target PPI network and an OP-related target PPI network to unravel the pharmacological mechanisms of XLGB against OP. Further, based on the plugin named CytoNCA, ${ }^{23} 343$ kernel targets were identified. We then input all kernel targets into the DAVID to enrich OP-associated pathways, and 31 pathways with $p \leq 0.05$ were obtained. They were performed using the OmicShare tools (http:// www.omicshare.com/tools) and are shown in - Fig. 4.

The 31 pathways containing 104 of the 343 targets might be the key pathways for the anti-OP mechanism of XLGB. Comparing the kernel targets of the pathway with the targets of 124 candidate compounds, we found that 19 kernel targets were overlapped (-Table 2 ), which might be considered as
A

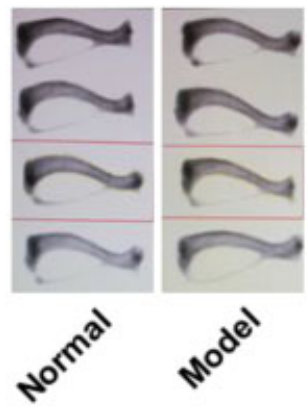

D

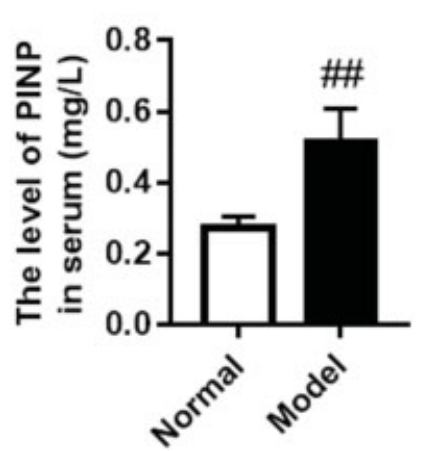

B

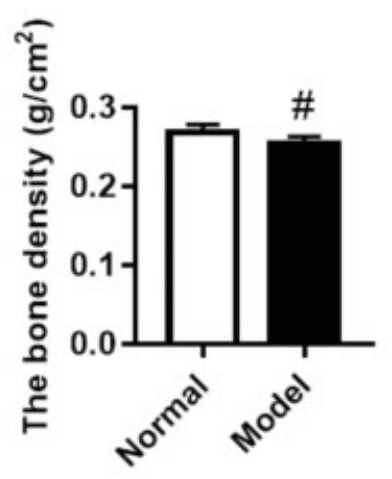

E

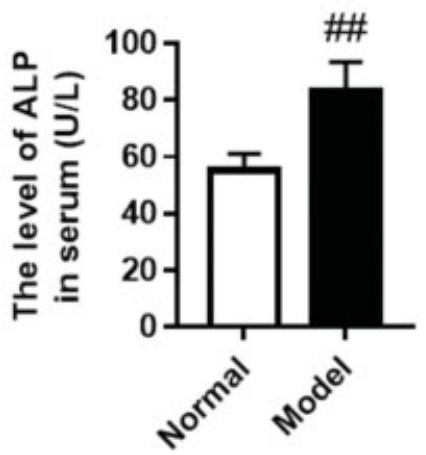

$\mathbf{F}$
C

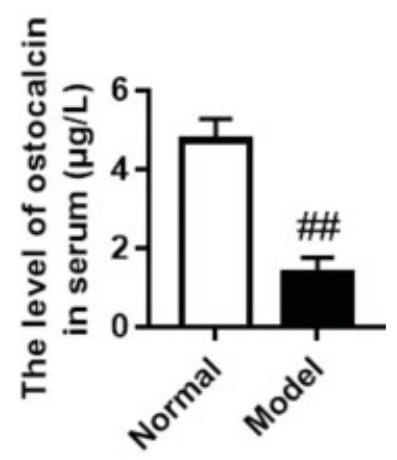

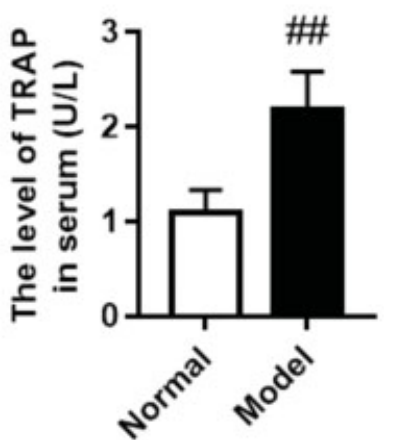

Fig. 2 Comparison of ( $A$ and $B$ ) bone density, and serum sample of (C) osteocalcin, (D) PINP, (E) ALP, and (F) TRAP between the model group and the normal control group. ${ }^{\#} p<0.05 ;{ }^{\# \#} p<0.01$ versus normal. ALP, alkaline phosphatase; PINP, propeptide of type I procollagen; TRAP, tartrate resistant acid phosphatase. 


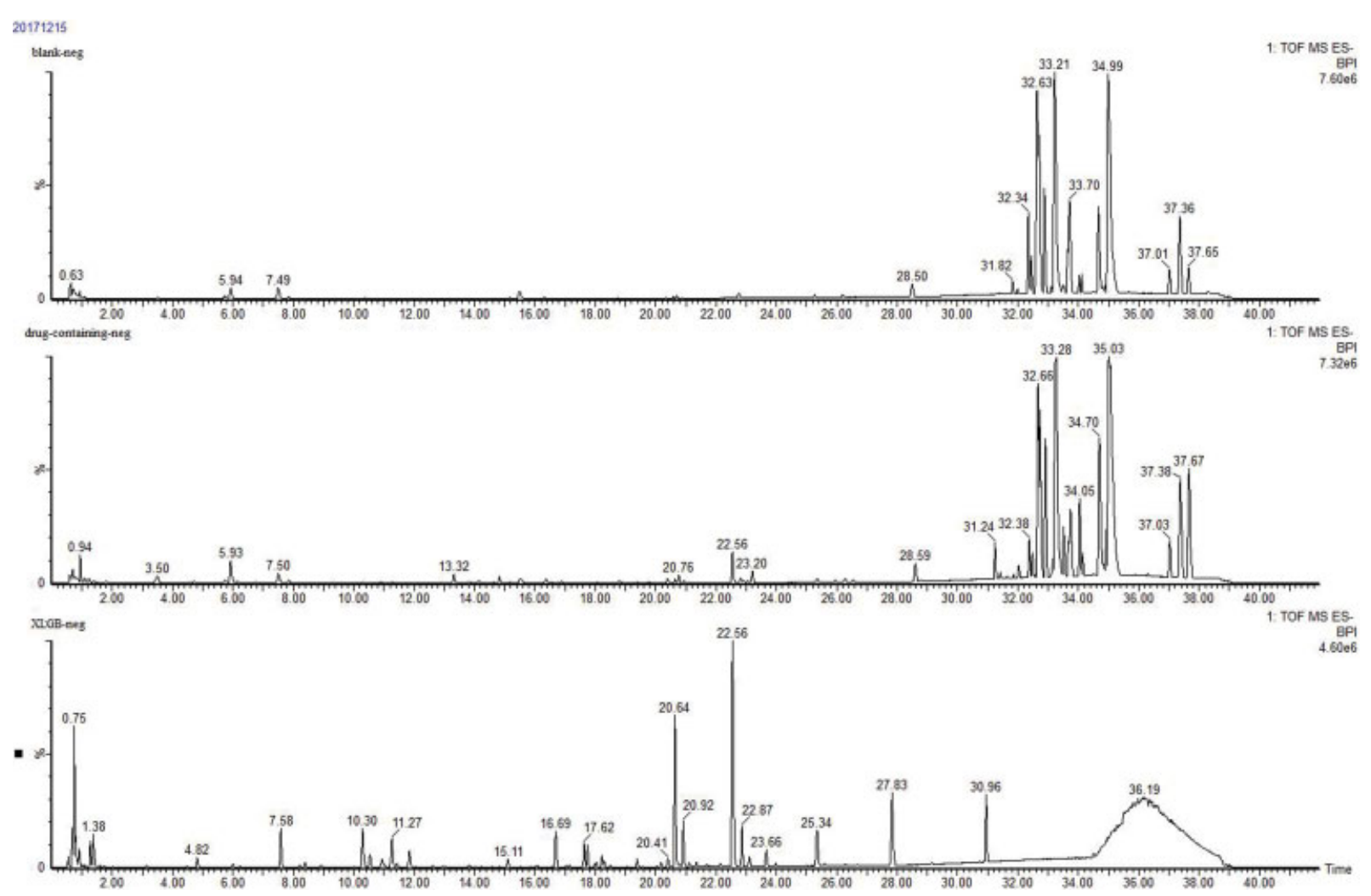

Fig. 3 LC-MS spectra demonstrating the pharmacochemistry analysis in the serum sample of OVX model of OP rat. LC-MS, liquid chromatography-mass spectrometry; OP, osteoporosis; OVX, ovariectomized.

potential markers and will function in the anti-OP process of XLGB.

Then, a compound-target-pathway network was constructed as shown in - Fig. 5, in which 105 compounds from six herbs of XLGB (- Supplementary Table S1, online only) acted on 28 pathways ( - Table 3 ) through 19 potential markers.

\section{Interaction between Potential Markers and Absorbed Compounds}

\section{Docking Results}

Molecular docking analysis was performed to investigate the interaction between the 22 absorbed compounds and 19 potential markers. Proteins without detected crystal structures were excluded from the analysis. Grid scores of the docking results are showed in - Supplementary Table $\mathbf{S 2}$ (online only). Grid scores less than $-80 \mathrm{kcal} / \mathrm{mol}$ were considered to be strong interaction. Our article suggested 14 potential markers with 13 absorbed components, among which 9 absorbed components were from Herba Epimedii, 3 absorbed components were from Radix Dipsaci, and 1 absorbed component was from Rhizoma Anemarrhenae.

\section{Absorbed Component-Potential Marker-Pathway Network}

A network was constructed to show the interaction among main absorbed components, potential markers, and pathways. -Fig. 6 demonstrates 13 absorbed components acted on 14 potential targets that connected to 28 pathways.

\section{Discussion}

It is well known that compounds will function on the premise that they are absorbed into the body. Thus, in this study, we investigated the interaction between screened potential markers and absorbed components to illuminate the anti-OP effect of XLGB in an in vivo study.

The clinical specification of XLGB is $0.5 \mathrm{~g} /$ piece following the instructions described. Adults take three pieces every time, twice a day. We first suggested an oral dose of 300 $\mathrm{mg} / \mathrm{kg} /$ day of XLGB for an OVX rat model of OP, which was equivalent to the corresponding clinical prescription dose for a $60 \mathrm{~kg}$ human subject and would make sure the results of our research study are as close as possible to clinical use. Then, we identified 22 main prototype components of XLGB in the serum sample of an OVX rat model of OP. This number was smaller than that given in Geng et al's report based on a healthy mice ${ }^{24}$ (57 prototype components). This may be resulted from animals in different conditions (normal and pathological). The TCM theory supports that the monarch and minister drugs may play a major role in a fufang prescription. In this article, we found that 11 of the 22 compounds are from Herba Epimedii (a monarch drug in the prescription), 7 from Radix Dipsaci, and 3 from Fructus Psoraleae, both of which are the minister drugs of XLGB.

The PPI networks were applied to investigate the relationship between compound targets and disease genes. We screened our potential markers based on network pharmacology analysis with PPI data and the enrichment analysis of KEGG pathways. We suggested a total of 124 candidate active 
Table 1 LC-MS data of 22 absorbed prototype compounds from OVX rat plasma after oral administration of XLGB

\begin{tabular}{|c|c|c|c|c|c|}
\hline$t_{\mathrm{R}} / \mathrm{min}$ & Selected ion & $\begin{array}{l}\text { Measured } \\
\text { mass }\end{array}$ & MS/MS fragmentation & Identification & Source \\
\hline 7.58 & {$[\mathrm{M}-\mathrm{H}]^{-}$} & 375.1281 & $\begin{array}{l}\text { 421.1342, 375.1281, } \\
213.0746\end{array}$ & Loganic acid ${ }^{b, c}$ & Radix Dipsaci \\
\hline 10.30 & {$[\mathrm{M}+\mathrm{HCOO}]^{-}$} & 403.1233 & $403.1233,197.8060$ & Sweroside ${ }^{b, c}$ & Radix Dipsaci \\
\hline 10.54 & {$\left[\mathrm{M}+\mathrm{HCOO}^{-}\right.$} & 435.1495 & 435.1495 & $\operatorname{Loganin}^{\mathrm{b}, \mathrm{c}}$ & Radix Dipsaci \\
\hline 11.03 & {$[\mathrm{M}+\mathrm{HCOO}]^{-}$} & 583.2044 & 583.2044 & Olivil-4"'-O-ß-D-glucopyranoside ${ }^{\mathrm{b}, \mathrm{c}}$ & Herba Epimedii \\
\hline 11.26 & {$[\mathrm{M}-\mathrm{H}]^{-}$} & 365.0866 & $365.0866,159.0431$ & Psoralenoside ${ }^{b}$ & Fructus Psoraleae \\
\hline 11.84 & {$[\mathrm{M}-\mathrm{H}]^{-}$} & 365.0872 & $365.0872,159.0436$ & Isopsoralenoside ${ }^{b}$ & Fructus Psoraleae \\
\hline 12.64 & {$[\mathrm{M}-\mathrm{H}]^{-}$} & 563.1424 & 563.1424 & Kaempferol 3-O-xylosyl-(1 $\rightarrow 2)$-rhamnoside ${ }^{\text {b }}$ & Herba Epimedii \\
\hline 17.76 & {$[\mathrm{M}-\mathrm{H}]^{-}$} & 823.2709 & $\begin{array}{l}\text { 823.2709, 661.2162, } \\
352.0941\end{array}$ & Diphylloside $A^{\text {b }}$ & Herba Epimedii \\
\hline 17.99 & {$[\mathrm{M}-\mathrm{H}]^{-}$} & 793.2575 & $793.2575,631.2067$ & 3''-O-Xylopyranosyl epimedoside $A^{b}$ & Herba Epimedii \\
\hline 18.22 & {$[\mathrm{M}-\mathrm{H}]^{-}$} & 807.2759 & $\begin{array}{l}\text { 807.2759, 645.2204, } \\
351.0852\end{array}$ & Diphylloside $\mathrm{B}^{\mathrm{b}}$ & Herba Epimedii \\
\hline 19.39 & {$[\mathrm{M}-\mathrm{H}]^{-}$} & 919.5035 & $\begin{array}{l}\text { 965.5030, 919.5035, } \\
\text { 757.4406, 595.3853 }\end{array}$ & Timosaponin B-II & $\begin{array}{l}\text { Rhizoma } \\
\text { Anemarrhenae }\end{array}$ \\
\hline 20.17 & {$[\mathrm{M}+\mathrm{HCOO}]^{-}$} & 883.2924 & $\begin{array}{l}\text { 883.2924, 675.2317, } \\
\text { 366.1105 }\end{array}$ & Epimedin $A^{a}$ & Herba Epimedii \\
\hline 20.41 & {$[\mathrm{M}+\mathrm{HCOO}]^{-}$} & 853.2797 & $\begin{array}{l}\text { 853.2797, 645.2215, } \\
\text { 366.1105 }\end{array}$ & Epimedin $\mathrm{B}^{\mathrm{a}}$ & Herba Epimedii \\
\hline 20.64 & {$\left[\mathrm{M}+\mathrm{HCOO}^{-}\right.$} & 867.2959 & $\begin{array}{l}\text { 867.2959, 857.2675, } \\
821.2942,659.2356, \\
366.1093\end{array}$ & Epimedin $\mathrm{C}^{\mathrm{a}}$ & Herba Epimedii \\
\hline 20.92 & {$[\mathrm{M}+\mathrm{HCOO}]^{-}$} & 721.2374 & $721.2374,367.1163$ & Icariin $^{\mathrm{a}}$ & Herba Epimedii \\
\hline 22.56 & {$[\mathrm{M}-\mathrm{H}]^{-}$} & 927.4996 & $\begin{array}{l}973.50481,927.4996 \\
603.39049,323.0965\end{array}$ & Asperosaponin $\mathrm{VI}^{\mathrm{a}}$ & Radix Dipsaci \\
\hline 25.34 & {$[\mathrm{M}-\mathrm{H}]^{-}$} & 795.4637 & $\begin{array}{l}\text { 841.4637, 795.4637, } \\
645.4028,471.3474\end{array}$ & Hederagenin $\beta$-sophorosyl ester ${ }^{b}$ & Radix Dipsaci \\
\hline 27.83 & {$[\mathrm{M}-\mathrm{H}]^{-}$} & 659.2362 & $\begin{array}{l}\text { 659.2362, 366.1090, } \\
351.0863\end{array}$ & 2"'-O-Rhamnosylicariside II ${ }^{\mathrm{a}}$ & Herba Epimedii \\
\hline 29.15 & {$[\mathrm{M}-\mathrm{H}]^{-}$} & 513.17767 & 513.17767 & Icariside $\|^{\mathrm{a}}$ & Herba Epimedii \\
\hline 29.53 & {$[\mathrm{M}-\mathrm{H}]^{-}$} & 911.50419 & $\begin{array}{l}\text { 957.5141, 911.50419, } \\
\text { 749.4536, 603. } 3957\end{array}$ & Akebia saponin $\mathrm{V}^{\mathrm{b}}$ & Radix Dipsaci \\
\hline 30.96 & {$\left[\mathrm{M}+\mathrm{HCOO}^{-}\right.$} & 603.3915 & $649.3969,603.3915$ & Akebia saponin $\mathrm{PA}^{\mathrm{b}}$ & Radix Dipsaci \\
\hline 37.04 & {$\left[\mathrm{M}+\mathrm{HCOO}^{-}\right.$} & 301.17961 & 301.17961 & Bakuchiol $^{\text {b }}$ & Fructus Psoraleae \\
\hline
\end{tabular}

Abbreviations: OVX, ovariectomized; $t_{R}$ : retention time; XLGB, Xianlinggubao capsule.

${ }^{\mathrm{a} C o n f i r m e d}$ with reference standards.

${ }^{\mathrm{b} C o n f i r m e d ~ w i t h ~ M S n ~ f r a g m e n t a t i o n . ~}$

${ }^{c}$ Confirmed with references. ${ }^{12}$

components of XLGB with 328 putative targets, among which 286 target genes were related to the development of OP. Enrichment analysis of KEGG pathway discovered 343 kernel targets and 31 potential pathways $(p<0.05)$, and 19 kernel targets from 28 pathways overlapped with the target compound, confirming their roles in anti-OP effect of XLGB. Then a network with 105 compounds, 19 targets, and 28 pathways was obtained, in which 105 compounds covered all six herbs of XLGB. However, in the compound-target-pathway network, only three compounds were identified in the plasma sample of OVX-OP rats, and this may be attributed to the very low dose of some compounds that could not be detected in this prescription. ${ }^{12}$
Moreover, molecular docking analysis showed 13 compounds (epimedin A, epimedin B, epimedin C, diphylloside B, diphylloside A, icariin, timosaponin B-II, asperosaponin VI, akebiasaponin $\mathrm{V}$, hederagenin- $\beta$-sophorosyl ester, olivil-4"O- $\beta$-D-glucopyranoside, 2"-O-rhamnosylicariside II, and 3"O-Xylopyranosyl epimedoside A) exhibited strong interactions with 14 potential markers (ABL1, CDK2, EGFR, HSP90AA1, HSP90AB1, HSP90B1, MAPK1, NFKB1, NFKB2, NOS2, RB1, SRC, TP53, and VCAM1). Nine of these compounds are from the monarch herb Herba Epimedii, three are from the assistant herb Radix Dipsaci, and one is from the minister herb Rhizoma Anemarrhenae. Our data suggested that the main active compounds in the monarch herbs are flavonoids 


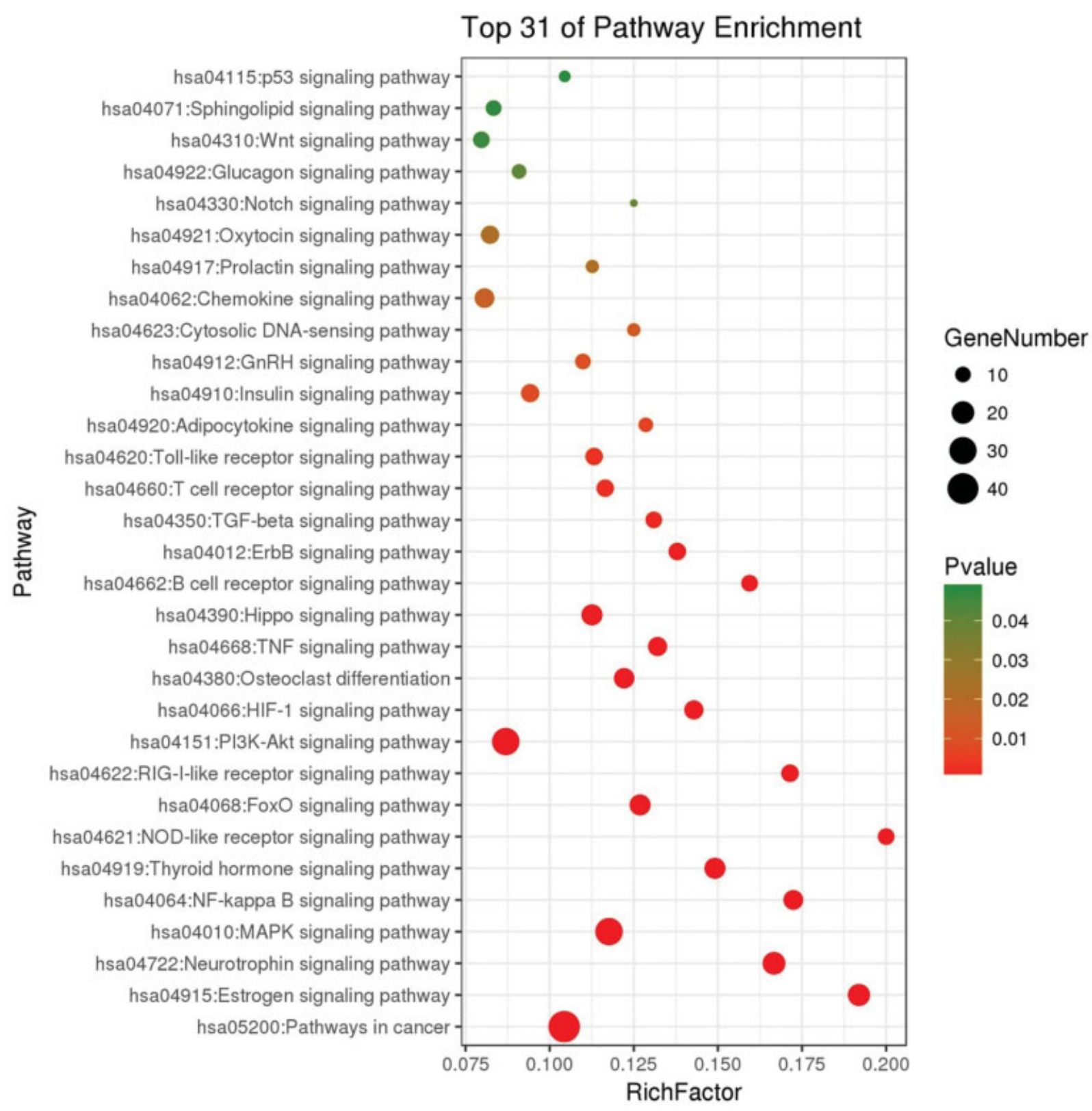

Fig. 431 OP-associated pathways with $p \leq 0.05$ through KEGG pathway enrichment. KEGG, Kyoto Encyclopedia of Genes and Genomes; OP, osteoporosis.

and saponins, which make up the most important therapeutic material basis in XLGB. As shown in the absorbed component-potential marker-pathway network, most of the compounds could regulate multiple signaling pathways via multiple targets. For example, epimedin A, epimedin B, and epimedin C (from Herba Epimedii) acted on NOS2, CDK2, EGFR, and MAPK1, among which MAPK1 and EGFR are kernel targets in this network, and connect with 22 and 9 signaling pathways, respectively. Timosaponin B-II (from Rhizoma Anemarrhenae) was linked to 10 targets and finally acted on 23 pathways. Icariin (from Herba Epimedii) connected with 21 pathways through four targets, and has been reported to promote osteogenesis through different pathways including EGFR, ${ }^{25} \mathrm{MAPK},{ }^{26}$ and $\mathrm{PI} 3 \mathrm{~K} / \mathrm{ATP}^{27}$ signaling pathways.
It is worth mentioning that most of the connected 28 pathways in the network were closely related to the development of OP, such as estrogen signaling pathway that usually underlies the anti-OP effect of many TCMs, 28,29 phosphorylation-mediated activation of the MAPK signaling pathway that plays a role in osteoblastic differentiation in mesenchymal cells, ${ }^{30,31}$ MAPK signaling molecules (ERK, p38, and JNK) that are related to osteoclast activation, differentiation, and survival, ${ }^{32,33}$ PI3K/ATP signaling that is associated with bone tissue metabolism, ${ }^{34,35}$ as well as HIF-1 signaling pathway, ${ }^{36}$ NF-KB signaling pathway, ${ }^{37}$ and Wnt signaling pathway. ${ }^{38}$ Some of them (for example the signaling pathway of PI3K-Akt, estrogen, MAPK, ErbB, NOD-like receptor, TNF, and neurotrophin) can be affected by more than five targets. 
Table 219 potential markers (overlapped kernel targets between the targets of the 31 pathways and the targets of 124 candidate compounds)

\begin{tabular}{|l|l|l|}
\hline NO. & Target name & Description \\
\hline 1 & ABL1 & Tyrosine-protein kinase ABL1 \\
\hline 2 & AR & Androgen receptor \\
\hline 3 & CDK1 & Cyclin-dependent kinase 1 \\
\hline 4 & CDK2 & Cyclin-dependent kinase 2 \\
\hline 5 & EGFR & Epidermal growth factor receptor \\
\hline 6 & ESR1 & Estrogen receptor 1 \\
\hline 7 & HSP90AA1 & Heat shock protein HSP 90- $\alpha$ \\
\hline 8 & HSP90AB1 & Heat shock protein HSP 90- $\beta$ \\
\hline 9 & HSP90B1 & Endoplasmin \\
\hline 10 & ICAM1 & Intercellular adhesion molecule 1 \\
\hline 11 & JUN & Transcription factor AP-1 \\
\hline 12 & MAPK1 & Mitogen-activated protein kinase 1 \\
\hline 13 & NFKB1 & Nuclear factor NF-kappa-B p105 subunit \\
\hline 14 & NFKB2 & Nuclear factor NF-kappa-B p100 subunit \\
\hline 15 & NOS2 & Nitric oxide synthase 2 \\
\hline 16 & RB1 & Retinoblastoma-associated protein \\
\hline 17 & SRC & Proto-oncogene tyrosine-protein kinase SrC \\
\hline 18 & TP53 & Cellular tumor antigen p53 \\
\hline 19 & VCAM1 & Vascular cell adhesion protein 1 \\
\hline
\end{tabular}

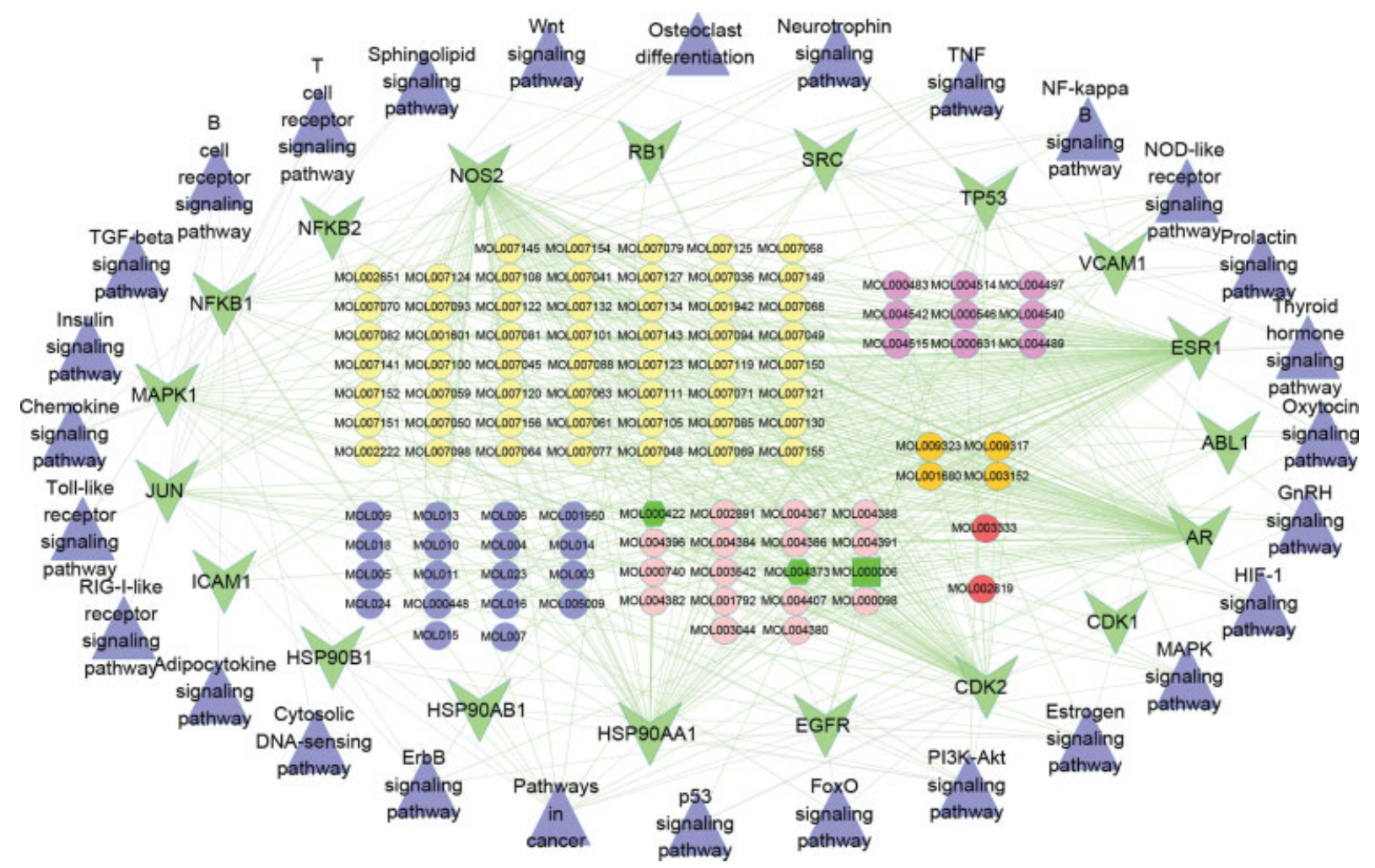

Fig. 5 Compound-target-pathway network (yellow, pink, blue, purple, orange, and red circles stand for compounds of Danshen, Yinyanghuo, Buguzhi, Zhimu, Xuduan, and Dihuang, respectively). Green V stands for compound targets. Blue triangle stands for pathways. Emerald green hexagon stands for common compounds of Zhimu and Yinyanghuo, Emerald green rectangle stands for common compounds of Danshen and Yinyanghuo. Gray lines stand for interaction among compounds and targets, and green lines stand for interaction among targets and pathways). 
Table 3 Screened 28 pathways based on KEGG pathway analysis which connected with 19 potential markers

\begin{tabular}{|c|c|c|c|}
\hline Pathway ID & Pathway description & $p$-Value & Kernel target genes \\
\hline hsa05200 & Pathways in cancer & $2.06 \mathrm{E}-08$ & $\begin{array}{l}\text { HSP90AB1, TRAF2, GRB2, PML, NFKBIA, NFKB1, NFKB2, PTEN, CTNNB1, AKT1, CUL2, } \\
\text { NOS2, TRAF6, MYC, CHUK, FN1, EGFR, AR, HSP90AA1, VHL, RELA, CREBBP, CBL, TP53, } \\
\text { SMAD3, SMAD2, RB1, CDK2, MAPK1, CDKN1A, HSP90B1, HDAC2, EP300, HDAC1, } \\
\text { JUN, NTRK1, IKBKG, MDM2, ABL1, IKBKB, CRK }\end{array}$ \\
\hline hsa04915 & $\begin{array}{l}\text { Estrogen } \\
\text { signaling pathway }\end{array}$ & $4.42 \mathrm{E}-08$ & $\begin{array}{l}\text { EGFR, HSP90AB1, HSP90AA1, GRB2, ESR1, HSPA1A, HSPA1B, SRC, HSPA1L, AKT1, } \\
\text { MAPK1, HSP90B1, SP1, JUN, CALM3, SHC1, HSPA8, CALM2, CALM1 }\end{array}$ \\
\hline hsa04722 & $\begin{array}{l}\text { Neurotrophin } \\
\text { signaling pathway }\end{array}$ & $1.86 \mathrm{E}-07$ & $\begin{array}{l}\text { GRB2, RELA, TP53, NFKBIA, NFKB1, YWHAE, AKT1, MAPK1, MAP3K3, JUN, NTRK1, } \\
\text { MAP3K1, CALM3, SHC1, IKBKB, ABL1, TRAF6, CRK, CALM2, CALM1 }\end{array}$ \\
\hline hsa04010 & $\begin{array}{l}\text { MAPK signaling } \\
\text { pathway }\end{array}$ & $2.11 \mathrm{E}-07$ & $\begin{array}{l}\text { TRAF2, GRB2, NFKB1, HSPA1A, HSPA1B, NFKB2, DAXX, HSPA1L, AKT1, TNFRSF1A, } \\
\text { MAP3K3, MAP3K1, TRAF6, MYC, HSPA8, CHUK, EGFR, RELA, TP53, FLNA, MAPK1, } \\
\text { ARRB2, ARRB1, NTRK1, JUN, IKBKG, HSPB1, MAP3K14, IKBKB, CRK }\end{array}$ \\
\hline hsa04064 & $\begin{array}{l}\text { NF-kappa B } \\
\text { signaling pathway }\end{array}$ & $6.54 \mathrm{E}-06$ & $\begin{array}{l}\text { ICAM1, TRAF2, RELA, NFKBIA, NFKB1, UBE2I, NFKB2, VCAM1, TNFRSF1A, CSNK2A1, } \\
\text { IKBKG, IKBKB, MAP3K14, TRAF6, CHUK }\end{array}$ \\
\hline hsa04919 & $\begin{array}{l}\text { Thyroid hormone } \\
\text { signaling pathway }\end{array}$ & $8.93 \mathrm{E}-06$ & $\begin{array}{l}\text { ACTB, KAT2B, CREBBP, TP53, ESR1, SRC, CTNNB1, AKT1, ACTG1, MAPK1, HDAC3, } \\
\text { EP300, HDAC2, HDAC1, MDM2, NCOR1, MYC }\end{array}$ \\
\hline hsa04621 & $\begin{array}{l}\text { NOD-like receptor } \\
\text { signaling pathway }\end{array}$ & $4.75 E-05$ & $\begin{array}{l}\text { HSP90AB1, MAPK1, HSP90B1, HSP90AA1, RELA, IKBKG, NFKBIA, NFKB1, TRAF6, } \\
\text { IKBKB, CHUK }\end{array}$ \\
\hline hsa04068 & FoxO signaling pathway & $6.91 \mathrm{E}-05$ & $\begin{array}{l}\text { EGFR, USP7, GRB2, CREBBP, SMAD3, SMAD2, PTEN, SIRT1, CDK2, AKT1, MAPK1, } \\
\text { PRMT1, CDKN1A, EP300, MDM2, IKBKB, CHUK }\end{array}$ \\
\hline hsa04622 & $\begin{array}{l}\text { RIG-I-like receptor } \\
\text { signaling pathway }\end{array}$ & $8.12 \mathrm{E}-05$ & $\begin{array}{l}\text { TRAF2, IKBKE, DDX3X, TBK1, RELA, MAP3K1, IKBKG, NFKBIA, NFKB1, TRAF6, IKBKB, } \\
\text { CHUK }\end{array}$ \\
\hline hsa04151 & $\begin{array}{l}\text { PI3K-Akt } \\
\text { signaling pathway }\end{array}$ & $8.50 \mathrm{E}-05$ & $\begin{array}{l}\text { HSP90AB1, YWHAZ, GRB2, NFKB1, PTEN, CDC37, AKT1, PPP2CA, MYC, CHUK, FN1, } \\
\text { EGFR, PPP2R1A, HSP90AA1, RELA, TP53, YWHAB, ITGA4, RPS6, YWHAE, BRCA1, } \\
\text { CDK2, MAPK1, CDKN1A, YWHAG, HSP90B1, IKBKG, YWHAQ, MDM2, IKBKB }\end{array}$ \\
\hline hsa04066 & HIF-1 signaling pathway & $1.14 \mathrm{E}-04$ & $\begin{array}{l}\text { EGFR, VHL, RELA, CREBBP, NFKB1, RPS6, AKT1, MAPK1, CUL2, CDKN1A, EP300, NOS2, } \\
\text { GAPDH, ENO1 }\end{array}$ \\
\hline hsa04380 & Osteoclast differentiation & $1.87 \mathrm{E}-04$ & $\begin{array}{l}\text { TRAF2, GRB2, RELA, NFKBIA, NFKB1, NFKB2, AKT1, MAPK1, TNFRSF1A, SQSTM1, JUN, } \\
\text { IKBKG, IKBKB, MAP3K14, TRAF6, CHUK }\end{array}$ \\
\hline hsa04668 & TNF signaling pathway & $2.53 \mathrm{E}-04$ & $\begin{array}{l}\text { ICAM1, TRAF2, RELA, NFKBIA, NFKB1, AKT1, VCAM1, MAPK1, TNFRSF1A, JUN, IKBKG, } \\
\text { IKBKB, MAP3K14, CHUK }\end{array}$ \\
\hline hsa04662 & $\begin{array}{l}\text { B cell receptor } \\
\text { signaling pathway }\end{array}$ & $3.39 E-04$ & AKT1, MAPK1, GRB2, JUN, RELA, CD81, IKBKG, NFKBIA, NFKB1, IKBKB, CHUK \\
\hline hsa04012 & ErbB signaling pathway & $5.80 \mathrm{E}-04$ & AKT1, EGFR, MAPK1, CDKN1A, GRB2, JUN, CBL, SHC1, ABL1, CRK, MYC, SRC \\
\hline hsa04350 & TGF- $\beta$ signaling pathway & $1.64 \mathrm{E}-03$ & $\begin{array}{l}\text { MAPK1, PPP2R1A, EP300, SP1, PPP2CA, CREBBP, SMAD3, SMAD2, SMURF1, MYC, } \\
\text { CUL1 }\end{array}$ \\
\hline hsa04660 & $\begin{array}{l}\text { T cell receptor } \\
\text { signaling pathway }\end{array}$ & $2.36 \mathrm{E}-03$ & AKT1, MAPK1, GRB2, JUN, RELA, CBL, IKBKG, NFKBIA, NFKB1, MAP3K14, IKBKB, CHUK \\
\hline hsa04620 & $\begin{array}{l}\text { Toll-like receptor } \\
\text { signaling pathway }\end{array}$ & $2.97 \mathrm{E}-03$ & AKT1, MAPK1, IKBKE, TBK1, JUN, RELA, IKBKG, NFKBIA, NFKB1, TRAF6, IKBKB, CHUK \\
\hline hsa04920 & $\begin{array}{l}\text { Adipocytokine } \\
\text { signaling pathway }\end{array}$ & $6.04 \mathrm{E}-03$ & AKT1, TRAF2, TNFRSF1A, RELA, IKBKG, NFKBIA, NFKB1, IKBKB, CHUK \\
\hline hsa04910 & Insulin signaling pathway & $8.20 \mathrm{E}-03$ & $\begin{array}{l}\text { AKT1, MAPK1, PPP1CA, GRB2, CBL, CALM3, SHC1, IKBKB, PPP1CC, RPS6, CRK, } \\
\text { CALM2, CALM1 }\end{array}$ \\
\hline hsa04912 & GnRH signaling pathway & $9.47 \mathrm{E}-03$ & EGFR, MAPK1, MAP3K3, GRB2, JUN, MAP3K1, CALM3, SRC, CALM2, CALM1 \\
\hline hsa04623 & $\begin{array}{l}\text { Cytosolic DNA-sensing } \\
\text { pathway }\end{array}$ & $1.26 \mathrm{E}-02$ & IKBKE, TBK1, RELA, IKBKG, NFKBIA, NFKB1, IKBKB, CHUK \\
\hline hsa04062 & $\begin{array}{l}\text { Chemokine } \\
\text { signaling pathway }\end{array}$ & $1.51 \mathrm{E}-02$ & $\begin{array}{l}\text { GRB2, RELA, NFKBIA, NFKB1, PXN, SRC, AKT1, MAPK1, ARRB2, ARRB1, IKBKG, SHC1, } \\
\text { IKBKB, CRK, CHUK }\end{array}$ \\
\hline hsa04917 & $\begin{array}{l}\text { Prolactin signaling } \\
\text { pathway }\end{array}$ & $2.14 \mathrm{E}-02$ & AKT1, MAPK1, GRB2, RELA, ESR1, NFKB1, SHC1, SRC \\
\hline hsa04921 & $\begin{array}{l}\text { Oxytocin signaling } \\
\text { pathway }\end{array}$ & $2.22 \mathrm{E}-02$ & $\begin{array}{l}\text { ACTB, EGFR, EEF2, PPP1CC, SRC, ACTG1, MAPK1, PPP1CA, CDKN1A, JUN, CALM3, } \\
\text { CALM2, CALM1 }\end{array}$ \\
\hline hsa04310 & Wnt signaling pathway & $4.60 \mathrm{E}-02$ & CSNK2A1, EP300, BTRC, JUN, CREBBP, TP53, RUVBL1, MYC, FBXW11, CUL1, CTNNB1 \\
\hline hsa04071 & $\begin{array}{l}\text { Sphingolipid } \\
\text { signaling pathway }\end{array}$ & $4.74 \mathrm{E}-02$ & AKT1, MAPK1, PPP2R1A, TRAF2, TNFRSF1A, PPP2CA, RELA, TP53, NFKB1, PTEN \\
\hline hsa04115 & p53 signaling pathway & $4.81 \mathrm{E}-02$ & CDK1, CDKN1A, TP53, MDM2, SFN, PTEN, CDK2 \\
\hline
\end{tabular}

Abbreviation: KEGG, Kyoto Encyclopedia of Genes and Genomes. 


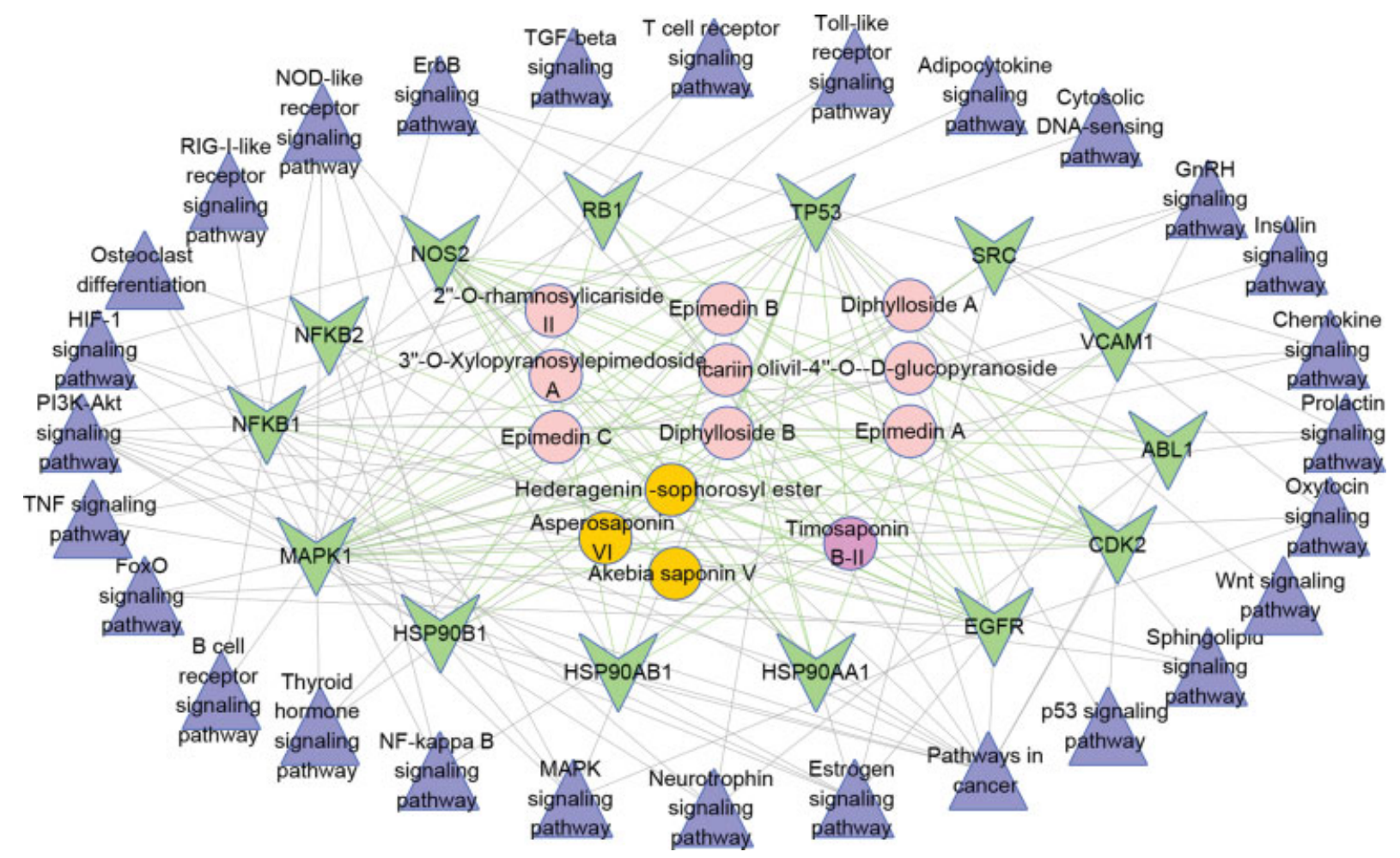

Fig. 6 Absorbed component-potential marker-pathway network (pink, purple, and orange circles stands for compounds of Yinyanghuo, Zhimu, Xuduan, respectively). Green $V$ stands for potential targets. Blue triangle stands for pathways. Gray lines stand for interaction between targets and pathways. Green lines stand for interaction between targets and compounds.

\section{Conclusions}

Our study was in accordance with traditional Chinese medicine theory and the multicomponent, multitarget, and multipathway characteristics of TCM. We investigated the possible active compounds of XLGB and the underlying therapeutic mechanisms from a systemic perspective. Our data provided a strong link between the major active components and potential targets, helped to delineate the anti-OP effect of XLGB and guide of the deconvolution of therapeutic effects of multi-component herbal treatment.

\section{Author Contributions}

Xiao Hu designed the experiments and checked the final manuscript. Yun-Hui Xu performed the experiments, analyzed and interpreted the results, and wrote the manuscript. Yi-Chun Sun performed some experiments, contributed materials, and checked the data. Jie Liu searched the relevant literature. Hui-Xin Li, Chun-Yue Huang, and Yuan-Yuan Pang provided helpful discussions. Tong Wu directed the research. All authors have read and approved the final version of this manuscript.

\section{Funding Statement}

This research was funded by the Project of Standardization of Chinese Materia Medica (Grant No. ZYBZH-C-GZ10), China Postdoctoral Science Foundation (Grant No. 2018M633022), and National Science and Technology Major Project (Grant No. 2018ZX09731-016).
Conflicts of Interest

The authors declare no conflict of interest.

\section{Acknowledgments}

We thank Guangzhou Yinfo Information Technology Co. Ltd. for providing an easy and versatile Dock Web App to aid the docking studies.

\section{References}

1 Awasthi H, Mani D, Singh D, Gupta A. The underlying pathophysiology and therapeutic approaches for osteoporosis. Med Res Rev 2018;38(06):2024-2057

2 Madrasi K, Li F, Kim MJ, et al. Regulatory perspectives in pharmacometric models of osteoporosis. J Clin Pharmacol 2018;58(05): 572-585

3 Burge R, Dawson-Hughes B, Solomon DH, Wong JB, King A, Tosteson A. Incidence and economic burden of osteoporosisrelated fractures in the United States, 2005-2025. J Bone Miner Res 2007;22(03):465-475

4 Chen P, Li Z, Hu Y. Prevalence of osteoporosis in China: a metaanalysis and systematic review. BMC Public Health 2016;16(01): 1039

5 Mithal A, Bansal B, Kyer CS, Ebeling P. The Asia-Pacific regional audit-epidemiology, costs, and burden of osteoporosis in India 2013: a report of International Osteoporosis Foundation. Indian J Endocrinol Metab 2014;18(04):449-454

6 Cooper C, Cole ZA, Holroyd CR, et al; IOF CSA Working Group on Fracture Epidemiology. Secular trends in the incidence of hip and other osteoporotic fractures. Osteoporos Int 2011;22(05):1277-1288

7 Liu K, Tan LJ, Wang P, et al. Functional relevance for associations between osteoporosis and genetic variants. PLoS One 2017;12 (04): 0174808 
8 Liu Y, Liu JP, Xia Y. Chinese herbal medicines for treating osteoporosis. Cochrane Database Syst Rev 2014;6(03):CD005467

9 Zhu HM, Qin L, Garnero P, et al. The first multicenter and randomized clinical trial of herbal Fufang for treatment of postmenopausal osteoporosis. Osteoporos Int 2012;23(04):1317-1327

10 Qin L, Zhang G, Hung WY, et al. Phytoestrogen-rich herb formula "XLGB" prevents OVX-induced deterioration of musculoskeletal tissues at the hip in old rats. J Bone Miner Metab 2005;23 (Suppl):55-61

11 State Food and Drug Administration of China Xianlinggubao Capsule, WS-10269 (ZD-0269)-2002. National Standards Assembly of Chinese Patent Medicine, 2002.

12 Dai Y, Tu FJ, Yao ZH, et al. Rapid identification of chemical constituents in traditional Chinese medicine fufang preparation xianling gubao capsule by LC-linear ion trap/Orbitrap mass spectrometry. Am J Chin Med 2013;41(05):1181-1198

13 Bao H, Guo H, Feng Z, Li X. Deciphering the underlying mechanism of Xianlinggubao capsule against osteoporosis by network pharmacology. BMC Complement Med Ther 2020;20(01):208

14 Lee AY, Park W, Kang TW, Cha MH, Chun JM. Network pharmacology-based prediction of active compounds and molecular targets in Yijin-Tang acting on hyperlipidaemia and atherosclerosis. J Ethnopharmacol 2018;221:151-159

15 Cheng M, Wang Q, Fan Y, et al. A traditional Chinese herbal preparation, Er-Zhi-Wan, prevent ovariectomy-induced osteoporosis in rats. J Ethnopharmacol 2011;138(02):279-285

16 Bahlous A, Kalai E, Hadj Salah M, Bouzid K, Zerelli L. Biochemical markers of bone remodeling: recent data of their applications in managing postmenopausal osteoporosis [in French]. Tunis Med 2006;84(11):751-757

17 Lei T, Liang Z, Li F, et al. Pulsed electromagnetic fields (PEMF) attenuate changes in vertebral bone mass, architecture and strength in ovariectomized mice. Bone 2018;108:10-19

18 Lipinski CA. Lead- and drug-like compounds: the rule-of-five revolution. Drug Discov Today Technol 2004;1(04):337-341

$19 \mathrm{Ru}$ J, Li P, Wang J, et al. TCMSP: a database of systems pharmacology for drug discovery from herbal medicines. J Cheminform 2014;6:13

20 Huang W, Sherman BT, Lempicki RA. Systematic and integrative analysis of large gene lists using DAVID bioinformatics resources. Nat Protoc 2009;4(01):44-57

21 Lang PT, Brozell SR, Mukherjee S, et al. DOCK 6: combining techniques to model RNA-small molecule complexes. RNA 2009;15(06):1219-1230

22 Missiuro PV, Liu K, Zou L, et al. Information flow analysis of interactome networks. PLOS Comput Biol 2009;5(04):e1000350

23 Tang Y, Li M, Wang J, Pan Y, Wu FX. CytoNCA: a cytoscape plugin for centrality analysis and evaluation of protein interaction networks. Biosystems 2015;127:67-72

24 Geng JL, Dai Y, Yao ZH, et al. Metabolites profile of Xian-Ling-GuBao capsule, a traditional Chinese medicine prescription, in rats by ultra performance liquid chromatography coupled with quadrupole time-of-flight tandem mass spectrometry analysis. J Pharm Biomed Anal 2014;96:90-103

25 Zhang D, Liu L, Jia Z, Yao X, Yang M. Flavonoids of Herba Epimedii stimulate osteogenic differentiation and suppress adipogenic differentiation of primary mesenchymal stem cells via estrogen receptor pathway. Pharm Biol 2016;54(06):954-963

$26 \mathrm{Wu} \mathrm{Y,} \mathrm{Xia} \mathrm{L,} \mathrm{Zhou} \mathrm{Y,} \mathrm{Xu} \mathrm{Y,} \mathrm{Jiang} \mathrm{X.} \mathrm{Icariin} \mathrm{induces} \mathrm{osteogenic}$ differentiation of bone mesenchymal stem cells in a MAPKdependent manner. Cell Prolif 2015;48(03):375-384

27 Zhai YK, Guo XY, Ge BF, et al. Icariin stimulates the osteogenic differentiation of rat bone marrow stromal cells via activating the PI3K-AKT-eNOS-NO-cGMP-PKG. Bone 2014;66:189-198

28 You L, Chen L, Pan L, Chen JY. New insights into the gene function of osteoporosis. Front Biosci 2013;18:1088-1097

29 Xu F, Ding Y, Guo Y, et al. Anti-osteoporosis effect of Epimedium via an estrogen-like mechanism based on a system-level approach. J Ethnopharmacol 2016;177:148-160

30 Higuchi C, Myoui A, Hashimoto N, et al. Continuous inhibition of MAPK signaling promotes the early osteoblastic differentiation and mineralization of the extracellular matrix. J Bone Miner Res 2002;17(10):1785-1794

31 Peng S, Zhou G, Luk KD, et al. Strontium promotes osteogenic differentiation of mesenchymal stem cells through the Ras/MAPK signaling pathway. Cell Physiol Biochem 2009;23(1-3):165-174

32 David JP, Sabapathy K, Hoffmann O, Idarraga MH, Wagner EF.JNK1 modulates osteoclastogenesis through both c-Jun phosphorylation-dependent and -independent mechanisms. J Cell Sci 2002; 115(Pt 22):4317-4325

33 Ikeda F, Nishimura R, Matsubara T, et al. Critical roles of c-Jun signaling in regulation of NFAT family and RANKL-regulated osteoclast differentiation. J Clin Invest 2004;114(04):475-484

34 Xi JC, Zang HY, Guo LX, et al. The PI3K/AKT cell signaling pathway is involved in regulation of osteoporosis. J Recept Signal Transduct Res 2015;35(06):640-645

$35 \mathrm{Ke} \mathrm{K}$, Li Q Yang X, et al. Asperosaponin VI promotes bone marrow stromal cell osteogenic differentiation through the PI3K/AKT signaling pathway in an osteoporosis model. Sci Rep 2016; 6:35233

36 Zhong H, Cao C, Yang J, Huang Q. Research on Relationship of HIF-1 Signaling Pathway and Postmenstrual Osteoporosis [in Chinese]. Sichuan Da Xue Xue Bao Yi Xue Ban 2017;48(06):862-868

37 Xu Q, Chen G, Liu X, Dai M, Zhang B. Icariin inhibits RANKLinduced osteoclastogenesis via modulation of the NF-KB and MAPK signaling pathways. Biochem Biophys Res Commun 2019;508(03):902-906

38 Amjadi-Moheb F, Akhavan-Niaki H. Wnt signaling pathway in osteoporosis: Epigenetic regulation, interaction with other signaling pathways, and therapeutic promises. J Cell Physiol 2019; 234(09):14641-14650 